\title{
Latest Developments in Corneal Surgery
}

\author{
An Expert Interview with Leopoldo Spadea
}

Policlinico Umberto I, Sapienza University of Rome, Italy

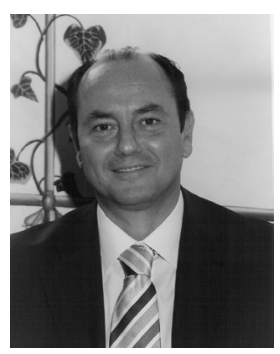

DOl: https://doi.org/10.17925/EOR.2018.12.1.17

\section{Leopoldo Spadea}

Leopoldo Spadea is an associate clinical professor in ophthalmology at the School of Medicine, Sapienza University of Rome, Italy. He is also Head of the Eye Clinic, Policlinico Umberto I Hospital, Rome, Italy. He has previously worked at the University of L'Aquila, Italy. He has presented papers at national and international congresses and symposia, and has published over 300 scientific publications. Having become an expert in refractive surgery in particular, he has been called upon to present official papers at many international courses and symposia. His interests include corneal topography, excimer laser refractive procedures (photo refractive keratectomy [PRK] and laser-assisted in situ keratomileusis [LASIK]), cataract surgery and keratoplasty.

\section{Keywords}

Big-bubble, deep anterior lamellar keratoplasty, keratoconus, microbubble technique

Disclosures: Leopoldo Spadea has nothing to disclose in relation to this article.

Review Process: This is an expert interview and as such has not undergone the journal's standard peer review process.

Authorship: All named authors meet the International Committee of Medical Journal Editors (ICMJE) criteria for authorship of this manuscript, take responsibility for the integrity of the work as a whole, and have given final approval to the version to be published.

open Access: This article is published under the Creative Commons Attribution Noncommercial License, which permits any noncommercial use, distribution, adaptation, and reproduction provided the original author(s) and source are given appropriate credit. () The Authors 2018.

Received: 20 July 2018

Published Online: 13 August 2018

Citation: European Ophthalmic Review. 2018; 12(1):17-18

Corresponding Author: Leopoldo Spadea,

Via Benozzo Gozzoli 34, 00142 Rome, Italy.

E: leopoldo.spadea@uniroma1.it

Support: No funding was received for the publication of this article.
K ratoconus is a disease characterised by progressive thinning, bulging and distortion of the cornea. Corneal transplantation (keratoplasty), is the standard of care for severe disease, but is associated with risks such as rejection of the transplanted cornea. Collagen cross-linking (CXL) is a relatively new conservative approach to strengthen corneal tissue by reforming new covalent bonds. In an expert interview, Leopoldo spadea of the University of Rome, Italy, discusses recent advances in keratoplasty and CXL.

\section{Q. Could you tell us a little about the microbubble technique in keratoplasty?}

The microbubble incision technique is a new surgical method which exploits the presence of small air bubbles in the premembrane layers. This removes deep stroma to reach Descemet membrane by means of manual layer-by-layer dissection. This technique is particularly useful in case of failure to establish a normal big-bubble using the Anwar big-bubble method. ${ }^{1}$ We know that deep lamellar anterior keratoplasty (DALK) has strict indications, for example in keratoconus, and provides better long-term results than penetrating keratoplasty, not so much in terms of visual acuity but in terms of survival of the transplant and reduction of the risk of rejection. In light of this, all the techniques that facilitate a more rapid and effective DALK dissection, in case of failure of the conventional big-bubble, are welcome. In particular, several preliminary studies seem to indicate microbubble technique as an alternative promising option for the surgeon. ${ }^{2}$

\section{Q. What are the limitations of this technique?}

Further studies with large cohorts of patients and long follow up are needed to confirm first impressions about the microbubble incision technique because it remains a technique that is difficult to standardise and is strongly dependent on the surgeon.

\section{Q. What are the advantages and limitations of corneal stromal demarcation line depth to assess collagen cross linking efficacy?}

Corneal stromal demarcation line $(\mathrm{DL})$ can be observed within the corneal stroma at 1 month post-operation. The interpretation of the depth of $\mathrm{DL}$ is certainly advantageous as it remains a reliable structural value and is now less operator-dependant thanks to the advent of recent optical coherence tomography devices which are able to easily detect the depth of DL. On the other hand, corneal stromal DL represents an indirect measurement of CXL penetration within the stroma. Current knowledge suggests that the 'the deeper, the better' principle is rather a simplistic approach for interpreting the clinical importance of the corneal stromal DL. ${ }^{3}$ However, we can be sure that $\mathrm{DL}$ remains one of the most reliable tools in evaluating efficacy of $\mathrm{CXL}$ treatment. 


\section{Q. What were the findings of your recent study comparing treatment protocols of corneal cross linking?}

I have dedicated my efforts in cross-linking treatments since development of this technique began several years ago. Over the years, several changes have been made, improving the effectiveness, safety and comfort for the patient. Based on my experience and my studies about the latest CXL protocols I can attest that, within certain limits, the accelerated protocol after corneal disepithelialisation (Epi-off $A-C X L$ ) remains the most effective in stabilising ectasia in corneas thicker than 400 microns. The key word of the present and the near future regarding CXL is 'customisation', such that we utilise a protocol that is tailored to the patient where possible not excluding the association of $\mathrm{CXL}$ with corneal regularisation methods, the so-called 'CXL plus'.
In thinner corneas, the safety parameters will guide us to one of the Epi-on treatments that can also be satisfactory in terms of effectiveness, although less so than the epi-off techniques.

\section{Q. What presentations are you most looking forward to at this year's European Society of} Cataract \& Refractive Surgeons (ESCRS) meeting?

In the next ESCRS meeting I am mainly looking forward to the novelties about refractive surgeries. Moreover, I will be interested to hear about laser-assisted surgery and corneal lamellar transplants, which have now almost completely supplanted penetrating techniques. Finally, I will be especially focused on the relatively recent Descemet membrane endothelial keratoplasty technique and the innovations concerning the topic of endothelial keratoplasty. $\square$

Anwar M, Teichmann KD. Big-bubble technique to bare Descemet's membrane in anterior lamellar keratoplasty. J Cataract Refract Surg. 2002:28:398-403.

Anwar M, Teichmann KD. Big-bubble technique to bare Descemet's membrane in anterior lamellar keratoplasty. J Cataract Refract Surg. 2002;28:398-403.

Spadea L, Di Genova L, Tonti E. Corneal stromal demarcation line after 4 protocols of corneal crosslinking in keratoconus determined with anterior segment optical coherence tomography. Spadea L, Di Genova L, Tonti E. Corneal Stor
J Cataract Refract Surg. 2018;44:596-602. 